\title{
Le paradoxe des émigrants indésirables pendant la monarchie de Juillet, ou les origines de 1 'émigration assistée vers l'Algérie
}

The Paradoxes of Undesirable Emigrants: The Origins of Assisted French

Emigration to Algeria during the July Monarchy.

Das Paradox der unerwünschten Auswanderer während der Julimonarchie oder die Ursprünge der unterstützten Auswanderung nach Algerien

\section{Jennifer Sessions}

\section{(2) OpenEdition}

\section{Journals}

Édition électronique

URL : https://journals.openedition.org/rh19/4045

DOI : $10.4000 /$ rh 19.4045

ISSN : $1777-5329$

Éditeur

La Société de 1848

Édition imprimée

Date de publication : 15 décembre 2010

Pagination : $63-80$

ISSN : 1265-1354

Référence électronique

Jennifer Sessions, «Le paradoxe des émigrants indésirables pendant la monarchie de Juillet, ou les origines de l'émigration assistée vers l'Algérie », Revue d'histoire du XIXe siècle [En ligne], 41 | 2010, mis en ligne le 30 décembre 2013, consulté le 21 septembre 2021. URL : http://journals.openedition.org/ rh19/4045; DOl : https://doi.org/10.4000/rh19.4045 


\section{JENNIFER SESSIONS}

\section{Le paradoxe des émigrants indésirables pendant la monarchie de Juillet, ou les origines de l'émigration assistée vers l'Algérie}

Avant même qu'Hussein Pacha, le dey d'Alger, ne capitule face au général de Bourmont, le 5 juillet 1830, l'invasion de la ville avait suscité en France des appels à la colonisation de la Régence d'Alger. Le sens du terme «colonisation" était à l'époque très fluctuant, marqué à la fois par les succès de la pensée libérale et par la mémoire douloureuse de la révolution haïtienne. En 1830, le terme "colonie» avait pour les Français, comme pour la plupart des Européens, des connotations très négatives en raison des représentations associées à la domination européenne dans le Nouveau Monde : la violence de la conquête qui entraîna l'extermination des populations indigènes, les lois commerciales restrictives, telles que l'Acte de navigation promulgué par les Britanniques en 1651 ou l'Exclusif, son équivalent français, et enfin l'asservissement des Amérindiens et des Africains, employés dans le développement des cultures de produits d'exportation comme le tabac, l'indigo, les épices et, surtout, le sucre. Pour les penseurs libéraux, adeptes du libre-échange et d'un marché du travail sans entraves, comme Jean-Baptiste Say, l'esclavage et les monopoles commerciaux étaient une hérésie, "un ordre de choses vicieux en soi, contraire aux intérêts et au bien-être d'un grand nombre d'hommes et que le progrès de l'espèce humaine doivent repousser tôt ou tard »' ${ }^{1}$. En réfléchissant à ce qu'il convenait de faire en Algérie, les économistes et les élites politiques se donnaient très souvent comme modèles les colonies fondées par les Grecs et les Romains ou encore par les Britanniques en Amérique du Nord $^{2}$. Ces références impliquaient des groupes de familles migrantes instal-

\footnotetext{
L'auteur tient à remercier Hélène Blais, Claire Fredj et Emmanuelle Saada, pour avoir relu cet article et aidé à sa traduction. p. 461.

1. Jean-Baptiste Say, Cours complet d'économie politique pratique, Paris, Rapilly, 1829, tome 4,

2. Après la Révolution, un consensus émerge : l'empire colonial de l'Ancien Régime est condamné alors que les vertus des empires grec, romain et britannique sont soulignées. Voir à ce propos Jennifer Sessions, Empire of Virtue: Colonialism and Political Culture in Nineteenth-Century France and Algeria, Ithaca, Cornell University Press, à paraître. Sur la transformation des représentations du projet impérial d'Ancien Régime et les débats post-révolutionnaires sur la colonisation, on se reportera à Anthony Pagden, Lords of All the World: Ideologies of Empire in Spain, Britian and France, c. 1500-c. 1800, New Haven, Yale University Press, 1995; Sankar Muthu, Enlightenment Against Empire, Princeton, Princeton University Press, 2003; Jennifer Pitts, A Turn to Empire: The Rise of Imperial Liberalism in Britain and
} 
lées de façon permanente sur des territoires conquis ou inhabités et qui, «à proprement parler [...] ne fondent pas même des colonies, mais agrandissent leur propre pays $»^{3}$. Selon eux, une colonie algérienne conforme à ce modèle serait au fondement d'un nouvel empire colonial, qui ne porterait pas la marque des crimes de la colonisation d'Ancien Régime et permettrait de soulager la pression démographique et économique pesant sur la société française.

La création d'une telle colonie en Algérie comportait des difficultés pratiques et idéologiques qui allaient devenir un vrai casse-tête pour les responsables politiques français pendant toute la période coloniale. Les Algériens firent en effet preuve d'une résistance militaire aussi farouche qu'imprévue, et l'administration militaire dut trouver un compromis entre les impératifs de la guerre de conquête et ceux de la colonisation civile. La résistance dirigée par Ahmed Bey de Constantine, Abd el-Kader et Bou Maza, ainsi que la complexité du régime foncier local et les malversations généralisées des Européens en matière de transaction foncière, entravèrent le transfert des terres aux colons. Parallèlement, les Français se montraient plutôt peu enclins à émigrer, et l'Algérie, comme la plupart des colonies de peuplement européennes d'alors, peinait à attirer les «bons» colons ${ }^{4}$. Confrontés à des flux de pauvres «indésirables» et de migrants issus de la classe ouvrière, les administrateurs coloniaux s'opposèrent à leurs homologues métropolitains sur la définition des objectifs de l'émigration et de la colonisation : s'agissait-il de fonder une société coloniale prospère ou simplement de servir les intérêts de la métropole? La colonisation avait-elle pour fonction de renforcer la domination militaire, ou bien au contraire la conquête devait-elle faciliter l'implantation européenne?

Les historiens de l'Algérie coloniale ont étudié les conflits engendrés par ces questions entre administrateurs militaires et civils en Afrique du Nord, en insistant sur leur impact dans l'élaboration de la «politique indigène» et

France, Princeton, Princeton University Press, 2005; Hélène Blais, "Qu’est-ce qu’Alger?” : le débat colonial sous la monarchie de Juillet", Romantisme, n 139, 2008-1, p. 19-32.

3. Jean-Baptiste Say, Cours complet d'économie politique pratique, Bruxelles, Société belge de librairie, 1840 (2 édition), p. 402.

4. L'historiographie sur le difficile recrutement des colons dans l'Empire britannique est très riche. Les travaux les plus récents sont : Robin Haines, Emigration and the Labouring Poor: Australian Recruitment in Britain and Ireland, 1831-1860, Londres, Macmillan, 1997; Lisa Chilton, Agents of Empire : British Female Emigration to Canada and Australia, 1860s-1930, Toronto, University of Toronto Press, 2007; James Belich, Replenishing the Earth: The Settler Revolution and the Rise of the Anglo-World, 17831939, Oxford, Oxford University Press, 2009. Dans le cas français, le recrutement des colons a été étudié par Yvette Katan, «Les colons de 1848 en Algérie : mythes et réalités», Revue d'histoire moderne et contemporaine, tome 31, 1984, p. 177-202; Isabelle Merle, Expériences coloniales : la Nouvelle-Calédonie, 1853-1920, Paris, Belin, 1995; Gille de Gantès, 'Migration to Indochina: Proof of the Popularity of Colonial Empire?' et Isabelle Merle ‘Drawing Settlers to New Caledonia: French Colonial Propaganda in the Late Nineteenth Century', in Tony Chafer et Amanda Sackur (ed.), Promoting the Colonial Idea: Propaganda and Visions of Empire in France, Houndsmills, Palgrave, 2002, p. 15-28 et p. 40-52; Leslie Choquette, Frenchmen into Peasants: Modernity and Tradition in the Peopling of French Canada, Cambridge (Mass.), Harvard University Press, 1997; Anne-Marie Medcalf, 'The Archives' Tales: Gender, Class and Colonial Migration Practices in Vietnam, 1897-1920', Intersections, no. 21, 2009, http:// intersections.anu.edu.au/issue $21 /$ medcalf.htm\#n4. 
sur les pratiques étatiques de contrôle des populations indigènes de l'Algérié. Le présent article infléchit cette perspective et examine comment des définitions contradictoires de la colonisation de peuplement se sont négociées de part et d'autre de la Méditerranée et se sont traduites dans les politiques d'émigration en Algérie. Les archives des services de l'État à Alger, à Paris et en province livrent les traces du développement d'une politique d'émigration assistée, et donc de pratiques de recrutement et d'identification des migrants. Leur analyse révèle cependant des divergences de points de vue, parmi les acteurs étatiques, sur la construction d'une colonie française en Algérie. Elle montre également l'emprise d'un État impérial qui cherche à identifier et à gérer non seulement les populations colonisées mais aussi les migrants coloniaux.

\section{L'Algérie ET LA COLONISATION DE PEUPLEMENT}

Les positions sur la colonisation algérienne au milieu du XIX ${ }^{\mathrm{e}}$ siècle peuvent être résumées par les propos de l'avocat et journaliste libéral, Eugène Lerminier, qui, en 1836, dans la Revue des Deux Mondes, avait lancé un appel pour une colonisation massive :

«Voici une vaste colonie sans esclaves qui s'offre à nous [...]. Là, nous n'avons besoin ni d'avilir, ni de tourmenter l'humanité. Là, le rotin du planteur ne frappera pas l'esclave devant la canne à sucre. Non, sur la terre d'Afrique tout peut se passer noblement; des hommes libres cultiveront la terre, les colons français et européens vivront par le travail sous la protection de nos armes, et la nouvelle colonie fera fleurir trois des plus nobles choses humaines, la liberté, l'agriculture et la guerre ${ }^{6}$.

La description de Lerminier sous-entend que les colons d'Algérie seraient l'antithèse des «aventuriers" égoïstes et mus par le seul appât du gain qui avaient fondé les colonies à esclaves. Pour les publicistes et les responsables politiques de l'époque, les colons d'Ancien Régime ne s'installaient aux colonies que le temps de faire fortune avant de rentrer en Europe profiter de leur bien mal acquis. Résidents temporaires, ces hommes ne s'intéressaient pas au développement économique et moral de leur région d'installation et formaient une société qui reposait sur le double fléau de l'esclavage et du monopole commercial. Au contraire les colons de l'Algérie pourraient devenir les habitants permanents d'une terre qu'ils allaient cultiver de leurs

5. Charles-André Julien, Histoire de l'Algérie contemporaine, tome 1, La conquête et les débuts de la colonisation (1827-1871), Paris, Presses universitaires de France, 1964, p. 335-336; Jacques Frémeaux, Les Bureaux arabes dans l'Algérie de la conquête, Paris, Denoël, 1993, p. 249-254; Osama Abi-Mershed, Apostles of Modernity: Saint-Simonians and the Civilizing Mission in Algeria, Stanford, Stanford University Press, 2010.

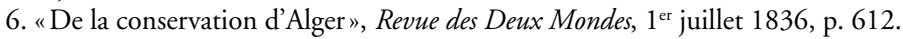


propres mains, formant une société stable permettant le développement de l'agriculture et de la vertu civique. Les seules manifestations de violence seraient le fait de l'armée se livrant au combat patriotique pour l'honneur et la prospérité de la France. La vision que Lerminier avait de la conquête algérienne entrait en complète contradiction avec les réalités de la guerre menée par l'armée d'Afrique, qui avait déjà suscité des critiques de journalistes, d'hommes politiques et d'officiers, dénonçant une atteinte aux normes de civilisation défendues par la France ${ }^{7}$. Il passait aussi sous silence le fait que l'agriculture coloniale dépendait de la mise au travail de la main d'œuvre algérienne, et ce de plus en plus au cours du XIX ${ }^{e}$ siècle, alors même que la guerre de conquête réduisait considérablement les productions indigènes, avec des conséquences désastreuses pour la population locale ${ }^{8}$. Mais son propos reprend une idée largement partagée par les publicistes, commentateurs et responsables politiques coloniaux du milieu du XIX ${ }^{\mathrm{e}}$ siècle, selon laquelle, comme Karl Marx allait plus tard l'affirmer, les «colonies réelles» pouvaient se définir comme "un sol vierge colonisé par des émigrants libres »".

L'appel à l'émigration de Lerminier met également en scène la figure idéale du colon supposé faire de la nouvelle possession, non pas une «colonie», mais une "province» de la France : «Partez donc, riches et capitalistes, allez; voilà d'honorables spéculations et des richesses pures. Appelez à vous les bras d'une pauvreté laborieuse, et faites à la France un riant et fertile jardin où ses enfants puissent aller chercher les uns l'abondance, les autres le repos, d'autres enfin la gloire. ${ }^{10}$ L'évocation ici d'une "pauvreté laborieuse» qui peut trouver «l'abondance» dans les richesses du sol algérien montre la convergence de l'argument impérialiste en faveur de la colonisation de peuplement et des préoccupations en matière de "question sociale», qui mobilisaient alors économistes, réformateurs et membres de l'administration. La littérature populaire et de nouvelles techniques statistiques participent alors au sentiment de crise imminente, de menace pour l'ordre social causée par les effets combinés de la surpopulation, de l'industrialisation naissante et de l'urbanisation. Les travailleurs, dont la rémunération avait grandement diminué, en raison de la mécanisation et de l'augmentation de la main-d'œuvre disponible, venaient s'entasser dans les grandes villes, nourrissant, aux yeux des élites bourgeoises inquiètes, les rangs des forces révolutionnaires ${ }^{11}$. Dans

7. Benjamin Brower, A Desert Named Peace: The Violence of France's Empire in the Algerian Sahara, 1833-1902, New York, Columbia University Press, 2009; Jennifer Sessions, 'Unfortunate Necessities: Violence and Civilization in the Conquest of Algeria', in Patricia Lorcin et Daniel Brewer (eds), France and Its Spaces of War: Experience, Memory, Image, London, Palgrave Macmillan, 2009, p. 29-44.

8. Tony Smith, 'The Causes of Muslim Impoverishment in Colonial Algeria', Revue de l'Occident musulman et de la Méditerranée, tome 17, no. 17, 1974, p. 151-153; Mahfoud Bennoune, The Making of Contemporary Algeria, 1830-1987, Cambridge, Cambridge University Press, 1988, p. 56-63; Diana Davis, Resurrecting the Granary of Rome: Environmental History and French Colonial Expansion in North Africa, Series in Ecology and History, Athens (Oh.), Ohio University Press, 2007, p. 32-34.

9. Karl Marx, Le Capital, trad. J. Roy, Paris, Maurice Lechâtre et Cie, 1872, p. 343, note 1.

10. Eugène Lerminier, "De la conservation....", loc. cit., p. 606.

11. Louis Chevalier, Classes laborieuses et classes dangereuses à Paris pendant la première moitié du XIXe 
ce contexte, l'émigration vers l'Afrique du Nord apparaissait non seulement comme une alternative aux défauts de l'empire d'Ancien Régime mais aussi comme une indispensable soupape de sécurité face aux pressions exercées sur la société métropolitaine. Selon l'un des premiers militants de la colonisation en l'Algérie, celle-ci représentait «un grand pas déjà vers l'extinction de la mendicité, disons même du paupérisme, et par suite une grande somme de sécurité pour l'ordre social; car alors, presque plus de vagabonds, ni de ces hommes oisifs et sans ressources, toujours prêts à se mettre à la solde des factieux contre la tranquillité publique ${ }^{12}$. De tels présupposés étaient partagés par des personnalités de sensibilités politiques très diverses, propriétaires conservateurs, comme le futur gouverneur général Thomas-Robert Bugeaud, ou romantiques socialistes - tels le saint-simonien Prosper Enfantin et le fouriériste Jules Duval - qui, tous, percevaient l'implantation en Algérie comme le moyen de transformer à la fois les sociétés métropolitaine et coloniale ${ }^{13}$.

Cependant, ce projet de colonisation de peuplement allait rencontrer d'importants obstacles. En dépit des incertitudes sur le maintien de la France en Afrique du Nord au début des années $1830^{14}$, l'une des premières préoccupations des autorités militaires fut d'identifier et de s'approprier les terres beylicales qu'elles imaginaient à l'origine de la richesse de la Régence ottomane. Usant du droit et de la force, les militaires confisquèrent progressivement des terres qu'ils assignèrent au domaine public dans l'idée de les redistribuer aux colons européens. Pourtant, cette appropriation systématique des terres représentait une contradiction idéologique de taille pour le régime bourgeois de la monarchie de Juillet, fondé sur le respect de la propriété privée ${ }^{15}$. En outre, la constitution d'un domaine public fut entravée par les opérations incessantes de la guerre de conquête, par la résistance armée des tribus à l'expropriation et par l'incompatibilité entre les conceptions algérienne et française du droit foncier et tout particulièrement de la propriété privée ${ }^{16}$. Comme l'observe Isabelle Grangaud, les propriétaires algériens devaient solliciter des témoins pour faire reconnaître leur titre de propriété et leur qualité d'usufruitier. Les Français, parce qu'ils ne connaissaient pas l'identité des

siècle, Paris, Plon, 1958; Frances Gouda, Poverty and Political Culture: The Rhetoric of Social Welfare in the Netherlands and France, 1815-1854, Lanham (Md.), Rowman et Littlefield, 1995; André Gueslin, Gens pauvres, pauvres gens dans la France du XIXe siècle, Paris, Aubier, 1988; Giovanna Procacci, Gouverner la misère : la question sociale en France (1789-1848), Paris, Seuil, 1993.

12. A. Amaury, Colonisation de l'Algérie. Observations, pour appendice et à l'appui d'un plan d'établissement en Algérie de Colonies Agricoles, proposé comme un d'entre les moyens les plus efficaces de parvenir à une diminution sensible de la mendicité et même du paupérisme en France, suivies du plan, établi sur une plus grande échelle, Paris, P.-F. Beaulé, 1842, p. 11.

13. Antony Thrall Sullivan, Thomas-Robert Bugeaud, France and Algeria, 1784-1849: Politics, Power, and the Good Society, Hamden (Conn.), Archon, 1983, p. 153-156; Saïd Almi, Urbanisme et colonisation : présence française en Algérie, Sprimont (Belg.), Mardaga, 2002, p. 29-31, 41-47.

14. Cette qualification a été introduite dans l'historiographie par les travaux essentiels de CharlesAndré Julien, Histoire de l'Algérie... op. cit., p. 64.

15. John Ruedy, Land Policy in Colonial Algeria: The Origins of the Rural Public Domain, Berkeley University of California Press, 1967, p. 37.

16. Ibidem. 
témoins ou mettaient en doute la véracité de leurs déclarations, ont bien souvent refusé de les reconnaître et ont saisi les biens en question, entravant la régularisation du régime foncier ${ }^{17}$.

La connaissance du pays et des populations était tout aussi nécessaire au succès de l'émigration européenne et, sur ce point également, les réalités locales ont ébranlé les tentatives étatiques de réglementation. Ces difficultés apparurent au grand jour dès les premiers mois de la conquête, quand le baron Baude, préfet de police de Paris et sous-secrétaire de l'Intérieur, avança le premier projet de colonisation officielle de l'ancienne Régence. Lors de l'hiver 1830-1831, particulièrement rigoureux, la capitale était agitée par des troubles provoqués par la crise économique et le procès des ex-ministres de Charles X. Des dizaines de grèves et d'attroupements de chômeurs se déroulèrent dans la capitale et, début janvier, les travailleurs qui réclamaient du pain et du travail occupent les ateliers de secours établis à Saint-Denis. Les forces de police ne pouvant maitriser la situation, la Garde Nationale fut envoyée pour disperser les manifestants. Responsable de la sécurité, Baude, dans un échange avec le ministre de l'Intérieur, suggéra qu'Alger pouvait offrir une solution permanente à ces troubles : «il n'y a peut-être de moyen de sortir de cette position pénible que d'élargir le débouché ouvert à une population inquiète et nécessiteuse; de faciliter les émigrations pour la côte d'Afrique ${ }^{18}$. Ce plan prévoyait d'envoyer à Alger jusqu'à 20000 Parisiens. Chaque "volontaire» devrait s'engager pendant cinq ans avant de recevoir une concession agricole. Ce plan, rapidement approuvé par le nouveau roi Louis-Philippe et par le Conseil d'État, fut mis à exécution. En une semaine, plus de 1700 travailleurs s'étaient inscrits à la mairie du $11^{\mathrm{e}}$ arrondissement et au milieu de l'été, quelque 4500 individus avaient été envoyés à Alger, aux frais de la ville de Paris et de la préfecture de la Seine. Il semble probable que les migrants parisiens cherchaient en Algérie ce qu'ils avaient perdu en France : le pain et le travail réclamés aux directeurs des ateliers de secours de Saint-Denis, qui fermèrent après les manifestations de janvier ${ }^{19}$. En octobre, le préfet de police notait une nette amélioration de l'ordre public qu'il attribua crânement à son programme d'émigration. Encouragé par ce résultat, il se fit l'avocat de la colonisation de masse en l'Algérie, moyen selon lui «indispensable» pour éviter les troubles et maintenir l'ordre public en France ${ }^{20}$.

Le projet de Baude apparut sous un jour très différent aux autorités françaises à Alger, qui ne virent dans les prétendus «volontaires parisiens » qu’une

17. Isabelle Grangaud, «Prouver par l'écriture : propriétaires algérois, conquérants français et historiens ottomanistes", Genèses, tome 74, ${ }^{\circ}$ 1, 2009, p. 38.

18. Arch. nat. (Archives nationales) $\mathrm{F}^{7} 3885$, Bulletin de Paris, 4 janvier 1831.

19. Alain Faure, «Mouvements populaires et mouvement ouvrier à Paris (1830-1834)", Le Mouvement social, tome 88, 1974, p. 73; David Pinkney, "Les ateliers de secours à Paris (1830-1831) précurseurs des ateliers nationaux de 1848", Revue d'histoire moderne et contemporaine, tome 12, 1965, p. $65-70$.

20. Arch. nat. F7 3885, Bulletin de Paris, 4 octobre 1831. 
charge financière et une source de désordre. Le général Berthezène, commandant en chef de l'armée d'Afrique, supplia le ministre de la Guerre de suspendre les convois de colons. Il affirmait avec force : "Ce serait une grande erreur et bien funeste de penser qu'il suffit de jeter sur les côtes d'Alger des familles indigentes prises au sein des villes ou dans les campagnes pour leur procurer du pain. [...] Il faut que les illusions cessent. $»^{21}$ Pour Berthezène, les terres de la campagne algéroise étaient en friche, et donc incultivables dans l'immédiat; les réserves de bois ne suffisaient pas aux besoins quotidiens et ne pouvaient a fortiori être utilisées pour la fabrication d'instruments agricoles. De plus, l'hostilité des populations locales rendait très précaires les conditions de vie des colons installés dans les concessions périphériques. Le secteur sous la responsabilité de Berthezène avait déjà été pris d'assaut par des "gens sans aveu" attirés par des rumeurs de colonisation. Il avait été obligé de les renvoyer en France, en faisant financer leur voyage par le budget de la colonie. Pour le général, l'armée n'avait établi son emprise que sur une portion limitée du territoire et il fallait attendre plusieurs années avant que la colonie ne pût fournir des moyens de subsistance à une population importante de migrants européens. Les envoyer prématurément en Algérie revenait à condamner des centaines d'individus à mourir de faim dans les rues d'Alger ou à devenir une charge pour l'administration locale ${ }^{22}$. Aux yeux du général, ces deux éventualités étaient, au fond, équivalentes : dans les deux cas, le citoyen en question renonçait à être responsable de sa situation et s'en remettait à l'État.

Les archives ne nous apprennent pas grand-chose du sort des "volontaires» parisiens. Le baron Pichon, premier intendant civil, a noté en 1833 qu'une centaine environ d'entre eux étaient décédés juste après avoir débarqué à Alger et que d'autres étaient rentrés en France sur des navires affrétés par le gouvernement ${ }^{23}$. Mais on ne sait ce qu'il est advenu de la majorité d'entre eux. Les conflits provoqués par leur arrivée à Alger ont toutefois soulevé des questions fondamentales à propos de la réalisation des projets de colonisation en Algérie et, au-delà, de l'émigration française en Afrique du Nord. Pour Baude, ainsi que pour l'administration municipale parisienne, pour les ministres, voire pour le roi, l'émigration coloniale était avant tout le moyen de débarrasser le royaume de ses sujets difficiles, qu'ils soient pauvres ou politiquement subversifs. De ce point de vue, la colonisation contribuait à l'ordre public et à la stabilité sociale de la métropole. Mais Berthezène se préoccupait avait tout de la sécurité et du budget du territoire sous sa responsabilité. Or, dans les conditions incertaines qui étaient celles de l'occupation, cette émigration de masse ne pouvait que représenter une charge insupporta-

21. SHD (Service historique de la Défense), 1H 7, lettre de Berthezène au ministre de la guerre, 21 mars 1831.

22. Ibidem.

23. Pichon, Alger sous la domination française : son état présent et son avenir, Paris, T. Barrois, 1833, p. $90-91$. 
ble pour l'administration locale. Elle aurait eu pour conséquence l'affaiblissement des fondations de la société coloniale, en y introduisant le malaise social qui rongeait la métropole. Toute politique d'émigration devait donc impérativement prendre en compte les conditions de vie en Afrique. Le cas des volontaires parisiens de Baude révèle les limites d'une analyse de l'émigration coloniale en Algérie à partir de l'alternative "émigration organisée» ou "émigration tolérée " ${ }^{24}$. Il suggère au contraire que le phénomène migratoire fut l'objet de compromis de part et d'autre de la Méditerranée, intégrant les impératifs de l'occupation militaire et ceux de l'enracinement colonial, les intérêts de la métropole et ceux des colons. Ces compromis se sont construits dans le contexte plus large d'une lutte violente entre les autorités militaires et les habitants de l'Algérie ottomane, ayant pour enjeu le territoire et la souveraineté. Les réponses des habitants de la Régence à l'invasion et à l'expropriation ont joué un rôle fondamental dans la forme qu'ont pris les flux migratoires qui ont fait de l'Algérie, avec la Nouvelle-Calédonie, la seule véritable colonie de peuplement dans le second empire colonial français.

\section{ENCADRER L'ÉMIGRATION COLONIALE}

Les premières années de l'occupation de l'Algérie sont, à juste titre, définies comme «une période d'incertitude» au cours de laquelle l'occupation - ses objectifs, son extension et même sa permanence dans le temps - restait en suspens. Ce n'est qu'à partir de 1838 que l'on peut réellement parler d'une politique de colonisation ou d'émigration. Cependant, les premières tentatives de contrôle de l'émigration vers l'Algérie qui commencent à cette époque révèlent les grandes lignes d'une logique de régulation qui perdure pendant toute la période du régime militaire en Algérie. Berthezène a vite été remplacé à la tête de l'armée d'occupation par le duc de Rovigo, mais sa critique d'une émigration incontrôlée et prématurée a laissé sa marque sur les politiques de ses successeurs. Tandis que le ministère de la Guerre organisait progressivement l'implantation européenne sur des concessions agricoles constituées à partir des terres confisquées aux Algériens, les responsables coloniaux et métropolitains ont cherché un moyen de sélectionner les candidats à l'émigration pour écarter de la colonie les individus indésirables et éviter les conflits entre Paris et Alger survenus à l'époque du préfet Baude.

L'attribution de passeports a constitué un premier outil pour identifier les candidats au départ et, le cas échéant, les catégoriser comme «indésirables » 25.

24. Emile Temime, «La migration européenne en Algérie au XIX ${ }^{\mathrm{e}}$ siècle : migration organisée ou tolérée?", Revue de l'Occident musulman et de la Méditerannée, tome 43, 1987, p. 31-45.

25. Les termes «désirables» et «indésirables» n'apparaissent pas dans les discours sur l'immigration avant les années 1920 : ils désignent alors les immigrants en France métropolitaine ( $c f$. Gérard Noiriel, Immigration, antisémitisme et racisme en France $\left(X I X^{e}-X X^{e}\right.$ siècle) : discours publics, humiliations privées, Paris, Fayard, 2007, p. 287 et $s q)$. Pourtant, je trouve utile de les utiliser ici parce qu'ils décrivent bien 
À cet égard, l'émigration coloniale doit être comprise autant dans la perspective de ce que Nancy Green et François Weil ont appelé « une gestion étatique de l'émigration" (l'élaboration de structures administratives pour contrôler des flux de population) que dans celle de l'ordre colonial ${ }^{26}$. Bien que la liberté de circulation, qui comprenait la libre sortie du territoire national, ait été l'une des libertés fondamentales établies par la Révolution, les personnes qui souhaitaient quitter leur canton de résidence devaient, comme sous l'Ancien Régime, obtenir un passeport des autorités locales. Cette condition, comme le faisaient remarquer les membres de l'administration, permettait le contrôle des criminels et des individus politiquement suspects et le bon fonctionnement des institutions de bienfaisance, organisées à l'époque au niveau local, en obligeant les "vagabonds» et "gens sans aveu» à résider dans la commune de leur domicile ${ }^{27}$. Pour empêcher les pauvres d'engorger les communes les plus généreuses, les autorités locales délivraient des passeports gratuits et une allocation de transport (secours de route) afin d'assurer le retour des indigents dans leurs communes d'origine. Malgré cela, il n'était pas rare que le ministère de l'Intérieur accuse les autorités locales de détourner l'usage des passeports et de ne voir «dans cette facilité [la possibilité de délivrer des passeports gratuits avec secours de route] qu'un moyen facile de débarrasser leurs communes de vagabonds et gens sans aveu, qui n'avaient aucun droit au bienfait de la mesure ${ }^{28}$. De même qu'en métropole les règles relatives aux passeports ont changé en fonction de l'évolution des représentations du «bon" et du "mauvais» citoyen, la réglementation relative à la délivrance des passeports permettant d'entrer en Algérie puis à l'octroi des passages libres vers la colonie, a évolué en suivant les transformations des catégories d'émigrant désirable et indésirable.

Les premières réglementations furent rédigées en mai 1831, alors même que les volontaires parisiens du préfet Baude partaient vers Alger. Le ministre de l'Intérieur, Casimir Perier, répondit aux protestations de Berthezène en transférant la prérogative de délivrance des passeports pour Alger du ministère des Affaires Étrangères aux préfectures, que l'on estimait mieux à même d'apprécier la situation et la qualité des migrants. Leur connaissance de la

l'ensemble des prises de position à l'égard des migrants vers l'Algérie dans la première période de la présence française.

26. Nancy Green et François Weil [dir.], Citoyenneté et émigration : les politiques du départ, Paris, Éditions de l'École des Hautes Études en Sciences Sociales, 2006, p. 11.

27. Daniel Nordman, "Sauf-conduits et passeports, en France, à la Renaissance», in Jean Céard et Jean-Claude Margolin [dir.], Voyager à la Renaissance : Actes du colloque de Tours 30 juin-13 juillet 1983, Paris, Maisonneuve et Larose, 1987, p. 145-58; Gérard Noiriel, «Surveiller les déplacements ou identifier les personnes? Contribution à l'histoire du passeport en France de la İ̀re à la III' République», Genèses, $\mathrm{n}^{\circ}$ 30, mars 1998, p. 77-100; John Torpey, The Invention of the Passport: Surveillance, Citizenship and the State, Cambridge, Cambridge University Press, 2000; Vincent Denis, "Administrer l'identité : le premier âge des papiers d'identité en France (XVIII'-milieu-XIX'e siècle) ", Labyrinthe, tome 5, 2000, http://labyrinthe.revues.org/index $258 . \mathrm{html}$.

28. Arch. dép. Var, 7M 5, ministère de l'Intérieur et des Cultes, division de la police générale, Circulaire $n^{\circ} 34$, «Nécessité d'arrêter l'abus des secours de route», 25 octobre 1833. 
population locale leur aurait en effet permis de «juger quelles sont les personnes qui présentent des garanties, sous le double rapport de la conduite et des moyens d'existence, et qui peuvent, sans inconvénient pour elles et pour le service en général, se diriger sur ce point de l'Afrique ${ }^{29}$. Selon cette circulaire de mai 1831, certaines catégories d'individus pouvaient être admises en Afrique du Nord sans créer de difficultés pour l'administration locale : les commerçants voyageant de manière ponctuelle pour leurs affaires, les individus souhaitant fonder des établissements agricoles et pouvant prouver qu'ils disposaient de ressources suffisantes pour acheter des terres, des outils et du bétail, ainsi que des «connaissances nécessaires pour former et faire prospérer leurs établissements». En aucun cas les préfets ne devaient accorder de passeports aux plus démunis ou aux travailleurs qui avaient «entendu parler de la colonisation d'Alger [et] demand[ai]ent d'y être envoyés [...] aux frais du Gouvernement ${ }^{30}$. Les préfets avaient pour devoir de convaincre ces derniers que l'Algérie ne pouvait leur offrir ni terre ni emploi, et que l'État ne leur accorderait aucun secours pour faciliter leur voyage ou leur installation. Une notice de juin 1831 publiée dans le Moniteur Universel indiquait l'intention du gouvernement de décourager «ceux qui croiraient améliorer leur position en se rendant en Afrique» et d'autoriser la migration des seuls individus "susceptibles de vivre du produit de leur travail, et de porter en Afrique quelques moyens d'existence ${ }^{31}$.

Ces premières régulations permettaient donc aux individus les plus fortunés de voyager librement entre la France et Alger et même de recevoir des aides sous la forme de passages gratuits sur les navires affrétés par le gouvernement, alors que les plus démunis étaient, autant que possible, écartés de la colonie naissante. Dans certains cas exceptionnels cependant, quand l'administration ou des entreprises privées avaient par exemple besoin de recourir à des ouvriers, ceux-ci pouvaient être autorisés à se rendre en Algérie. À cet égard, le régime des passeports algériens suivait la logique de l'institution des passeports pour l'intérieur en France : il s'agissait de protéger les grandes villes, notamment Paris, d'une "population flottante» de vagabonds, mendiants et autres indigents. La circulaire spécifiait d'ailleurs que les passeports des voyageurs pour l'Algérie devaient être assimilés à des passeports pour l'intérieur et non plus aux documents nécessaires pour quitter le territoire français.

C'est seulement en 1838 que les dispositions de la circulaire de 1831 furent modifiées, après la signature du traité de Tafna, qui permit un apaisement provisoire en Algérie. À cette époque, l'extension du territoire sous contrôle français et l'établissement permanent de militaires pour en assurer

29. Arch. dép. Corrèze 6M 400, circulaire du ministre de l'Intérieur, 18 mai 1831.

30. Ibidem.

31. Archives nationales d'Outre-Mer (Arch. nat. Outre-Mer), Afrique IV/5, extrait du Moniteur du mardi 21 juin 1831 . 
la défense nécessitèrent l'augmentation du flux d'émigrants, en particulier de cultivateurs, pour exploiter les terres récemment conquises, et d'ouvriers qualifiés pour participer aux grands chantiers de travaux publics destinés à mettre en valeur la colonie et à protéger les colons. En août 1838, le ministre de l'Intérieur déclara que «le moment était venu de diminuer la rigueur des mesures de précaution encore observées relativement au passage en Afrique des ouvriers sans occupation en Afrique »32. La délivrance des passeports ne relèverait plus des seules prérogatives préfectorales, et la compétence fut étendue aux sous-préfets et aux maires. Le critère financier fut assoupli pour permettre à tous les travailleurs valides de se rendre librement dans la colonie. Les émigrés devaient présenter des certificats de santé et de moralité afin d'obtenir leur passeport, mais la catégorie d'indésirable était désormais réduite aux individus jugés incapables de travailler (invalides, personnes âgées et enfants âgés de moins de seize ans) ou peu disposés à le faire (vagabonds, récidivistes, ou tout individu sans profession clairement identifiable). Afin de faciliter l'émigration des travailleurs "réellement utiles", l'État prenait maintenant en charge le passage des travailleurs qualifiés du secteur agricole et du bâtiment ainsi que des agriculteurs ayant droit à des concessions agricoles $^{33}$. Les préfets étaient enfin autorisés à financer, sur le budget départemental de l'assistance, des secours de route couvrant les frais de transport des migrants, depuis leur domicile jusqu'à Toulon, d'où ils embarquaient ensuite pour Alger.

Ces mesures présentaient un tournant majeur dans la politique d'émigration et relancèrent le conflit entre Alger et Paris. Le comte Guyot, nouvel intendant civil à Alger, commença à émettre des réserves sur le nombre et la qualification des nouveaux migrants, pour la plupart des artisans certes qualifiés mais urbains, incapables donc de s'installer comme fermiers ou de trouver à s'employer dans des chantiers de travaux publics exigeant soit le seul usage de la force physique soit une connaissance de métiers du bâtiment très spécifiques. Une fois encore, l'administration coloniale fut obligée de fournir des secours à des centaines de pauvres, émigrés désormais chômeurs et incapables de s'adapter au marché local de l'emploi. Le ministre de la Guerre demanda à l'administration algérienne de faire correspondre la demande de travail aux "besoins des ouvriers arrivants" : "Il ne faut pas que des ouvriers valides puissent à bon droit prétendre qu'ils ont offert leurs bras et qu'ils ont été refusés. ${ }^{34}$

Ces instructions n'apaisèrent guère les tensions, qui se poursuivent jusqu'à la reprise des hostilités avec Abd el-Kader. Ensuite, les résultats catastrophiques de la nouvelle politique d'émigration convainquirent le nouveau minis-

32. Arch. nat. Outre-Mer, F80 1793, circulaire du ministre de l'Intérieur, 30 août 1838.

33. Ibidem. 1838 .

34. Arch. nat. Outre-Mer, F80 1793, ministre de la Guerre à l'intendant civil d'Alger, 7 novembre 
tre de la Guerre, le général Despans-Cubières, de ralentir le rythme effréné des passages gratuits - le ministère avait accordé près de 4000 permis dans les neuf mois suivant la promulgation du circulaire du mois d'août 1838 et d'agir en collaboration plus étroite avec le gouverneur général Valée pour déterminer le nombre de travailleurs et le type de compétences requis dans la colonie. Au cours des années 1839 et 1840, avec le maréchal Soult exerçant les fonctions de président du Conseil et de ministre de la Guerre, les deux administrations développèrent une réglementation plus cohérente de délivrance des passeports et d'attribution des passages gratuits, objet d'une directive commune en mai 1839 : sous-préfets et maires devaient dorénavant refuser de délivrer des passeports aux travailleurs et aux cultivateurs qui n'avaient pas préalablement obtenu du ministère un permis de passage gratuit ${ }^{35}$. Le nombre de ces passages serait déterminé en fonction des besoins du marché du travail d'Alger, estimés par les fonctionnaires coloniaux. Les directeurs des divers services coloniaux informeraient des besoins en main d'œuvre le gouverneur général, qui transmettrait au ministère de la Guerre, tenant lui-même le registre des demandes d'aide à l'émigration transmises par les préfets et les maires. Il accorderait des passages gratuits aux individus exerçant les métiers dont on avait besoin en Algérie, selon l'ordre d'enregistrement de leur demande. La liste des professions recherchées avait été raccourcie et ne mentionnait plus que quelques métiers du bâtiment, le plus souvent ne requérant aucune qualification, comme celui de terrassier ou de manœuvre pour la construction de routes. Les familles « reconnues capables de se livrer à la culture» et disposant de ressources suffisantes pour subvenir à leurs besoins jusqu'à la première récolte pouvaient demander des concessions d'une surface maximale de douze hectares. Mais priorité était donnée aux hommes jeunes et célibataires «en raison de la nécessité de se transporter fréquemment d'un point sur l'autre $»^{36}$.

Les archives nous permettent de décrire les pratiques engendrées par ce dispositif, compromis entre la volonté du gouvernement métropolitain d'accroître l'émigration et les réticences de l'administration coloniale à l'égard d'une immigration dont le contrôle lui échapperait. Ces impératifs contradictoires dessinent le portrait d'un migrant "désirable», en bonne santé, de bonne moralité et doté de qualifications professionnelles. Ils permettent en outre de comprendre que les colons-concessionnaires et les colons-ouvriers étaient soumis à des critères moins stricts. L'utilisation de dossiers imprimés à chaque étape du recrutement est le signe d'une bureaucratisation de l'émigration vers l'Algérie et du caractère dorénavant routinier de la procédure de contrôle. Le bureau de l'Algérie au ministère de la Guerre commença à

35. Arch. nat. Outre-Mer, F80 1793, circulaire du ministre de la Guerre, 10 mai 1839.

36. Arch. nat. Outre-Mer, F80 1793, circulaire du ministre de la Guerre, 28 février 1841 ; Arch. dép. Haut-Rhin 6M 354, circulaire du ministre de la Guerre, 19 novembre 1840. Le montant requis des familles concessionnaires est fixé à 1200 francs. 
faire imprimer une lettre-type accompagnant les permis de passage gratuit, envoyée aux administrations locales (préfectures et mairies) pour transmission aux intéressés. Dans les régions où l'émigration était particulièrement importante, les administrations firent également imprimer des formulaires et notamment des passeports précisant l'itinéraire obligatoire pour les récipiendaires des secours de route, et des lettres informant les maires de la transmission des avis de passage. Dans certaines villes, comme Strasbourg et Nantes, ce processus de bureaucratisation est perceptible même au niveau municipal, puisque les commissaires de police chargés de vérifier la situation des demandeurs de passage gratuit avaient eux aussi des formulaires imprimés à leur disposition.

De même que l'État s'efforçait de contrôler les mouvements de population à l'intérieur du territoire français, les autorités locales ont joué un rôle fondamental dans la gestion des flux vers l'Algérie. Les ministres de la Guerre et de l'Intérieur ont confié aux préfets la responsabilité de distinguer les "bons" migrants des "indésirables", et les préfets se sont acquittés de cette tâche en demandant à des fonctionnaires plus proches de la population (sous-préfets, maires et commissaires de police) de vérifier l'identité des individus ayant déposé des demandes de passeport ou de passage gratuit et d'enquêter sur leur situation financière, morale et professionnelle. Comme ces fonctionnaires étaient censés connaître personnellement leurs administrés, on attendait d'eux des informations fiables. Cette proximité avec les populations leur a aussi valu d'être sollicités pour participer aux efforts de promotion de l'émigration quand la situation en Algérie exigea un recrutement plus massif. Un véritable matériel de propagande fut envoyé aux préfets et aux maires qui firent publier des articles dans la presse locale, afficher des placards sur les bâtiments publics et distribuer des livrets d'information rédigés par le ministère de la Guerre. Pour les candidats à l'émigration, ces fonctionnaires jouaient le rôle d'intermédiaires dans leurs démarches auprès des préfets ou du ministère pour obtenir un passeport et un passage gratuit. Dans les communes rurales, le maire était souvent mis à contribution par des candidats illettrés pour remplir les dossiers de demande de passage gratuit, de passeport ou de secours de route pour couvrir les frais de transport jusqu'à Marseille ou Toulon.

En pratique, l'importance donnée à l'administration locale s'est révélée être à la fois un atout et une difficulté pour le contrôle administratif de l'émigration coloniale, dans la mesure où ces fonctionnaires profitaient de leur connaissance des candidats à l'émigration pour faire avancer ou au contraire détourner les objectifs de leurs supérieurs hiérarchiques. Certains représentants locaux de l'État auraient fait un usage abusif du système des secours de route dans sa version métropolitaine - accusations qui se retrouvent dans les critiques émises par les bureaux ministériels et préfectoraux. Les fonctionnaires locaux, selon le ministre de la Guerre et le gouverneur Général, auraient 
systématiquement exagéré les ressources financières des postulants, dissimulé leurs tares physiques ou franchement menti sur leur moralité, pour favoriser certains candidats ou se débarrasser d'individus jugés corrompus, inutiles ou dangereux. Ces accusations n'étaient peut-être pas toutes sans fondement. Une note, dans les archives municipales de Strasbourg, demandant au commissaire de police de fournir au dénommé Charles Mülbach un certificat de moralité - afin "de débarrasser la ville de ce jeune homme, qui a déjà été arrêté deux fois pour mendicité» - n'est sans doute qu'un exemple particulièrement explicite de telles pratiques, de la part de fonctionnaires qui avaient depuis longtemps l'habitude de contourner les dispositions en matière de passeports pour l'intérieur ${ }^{37}$. Les préfectures semblent elles aussi avoir régulièrement émis des passeports pour l'Algérie pour des individus dont la situation ne correspondait pas aux critères exigés du ministère, voire, dans certains cas, répandu de fausses rumeurs sur les aides fournies aux émigrants afin de favoriser les départs. Inversement, dans des périodes où le ministère cherchait à développer l'émigration, les représentants locaux de l'Etat, préoccupés par l'impact de ces départs sur le marché du travail local, s'employaient à contrecarrer ces efforts en ne faisant pas connaître les aides auxquelles pouvaient prétendre les émigrants ou en diffusant des informations décourageantes sur les conditions de vie dans la colonie ${ }^{38}$.

Les tensions entre l'État métropolitain, ses services décentralisés et l'administration coloniale en Algérie sur la question de l'émigration reflètent le paradoxe au cœur de tout projet de fondation d'une colonie de peuplement : les fonctionnaires à tous les niveaux sont alors en situation de se disputer des populations qu'ils considèrent désirables sur le plan social, moral et économique. Les conflits occasionnés par les migrations de Parisiens en 1831 sont assez représentatifs des débats autour de la colonisation de peuplement dans les «Néo-Europes» du XIX siècle. Les autorités britanniques par exemple se trouvaient dans cette période en conflit ouvert avec l'Australie, le Canada, la colonie du Cap et les Etats-Unis à propos de la "qualité» des émigrants assistés en provenance des îles britanniques ${ }^{39}$. On peut noter toutefois que les responsables politiques français semblent avoir tout ignoré des moyens employés par les Britanniques pour résoudre ces tensions. Les conflits entre administration centrale et fonctionnaires locaux trouvent également de nombreux échos dans l'histoire de l'État français au XIX siècle. Les remarques de Roger Price sur le rôle ambigu des maires dans la répression des soulèvements populaires du XIX ${ }^{e}$ siècle, par exemple, pourraient très bien s'appli-

37. Arch. mun. (Archives municipales) de Strasbourg, $271 \mathrm{MW} 67$, note jointe au gérant de la Maison de Refuge, 23 novembre 1847.

38. Arch. dép. Var, 11M 3/4, préfet du Var au ministre de l'Intérieur, 2 juin 1847.

39. Aristide Zolberg, «La Révolution des départs», in Nancy Green et François Weil [dir.], Citoyenneté et émigration..., op. cit., p. 37-64; Robin Haines, 'Indigent Misfits or Shrewd Operators? Government-Assisted Emigrants from the United Kingdom to Australia, 1831-1860', Population Studies tome $48, \mathrm{n}^{\circ} 2,1994$, p. $223-247$. 
quer à leur implication dans la fondation d'une colonie de peuplement en Afrique : «Parfois, ils se sont comportés moins comme les représentants du gouvernement que comme les défenseurs des intérêts de leur commune de résidence $»^{40}$.

À certains égards, ces similarités peuvent indiquer que l'encadrement de l'émigration coloniale a été l'un des éléments d'un processus plus large de consolidation de l'État et de développement de pratiques administratives de contrôle de la population. Mais on peut dégager deux autres conclusions de cette analyse succincte de l'encadrement de l'émigration vers l'Algérie dans les dix premières années de la conquête. D'une part, il existe une continuité frappante dans la manière dont l'administration se représente les migrants et tout particulièrement les plus pauvres d'entre eux. Même si, à partir des années 1840, avec le développement des ouvrages de travaux publics, les travailleurs entrent dans la catégorie de "migrants désirables", l'opposition entre «désirable» et «indésirable» reste au fondement de la politique d'émigration - que ses objectifs soient d'intensifier ou au contraire de ralentir les flux vers l'Algérie. Avec le temps, les administrations coloniale et métropolitaine ont rejoint le point de vue exprimé par le Général Berthezène en 1831. Un quart de siècle plus tard, le ministre de la Guerre lui-même estimait qu'il fallait à tout prix éviter «la venue en Algérie de familles nécessiteuses qui ne rendent aucun service à la colonisation et retombent presque toujours à la charge des administrations locales $»^{41}$. En revanche, il convenait de faciliter les démarches du "colon sérieux», doté de suffisamment de ressources pour prendre en charge les frais d'installation sur une terre acquise par la force.

D'autre part, l'idéal affirmé à l'époque de la colonisation agricole n'a que très partiellement correspondu aux besoins réels d'un projet de peuplement. La plupart des 20000 individus qui s'étaient vu accorder un passage gratuit entre 1841 et 1845 venaient d'un milieu urbain et exerçaient des métiers artisanaux. Les analyses quantitatives faites à partir des registres tenus par le ministère de la Guerre pendant cette période révèlent non seulement que les trois quarts des bénéficiaires des passages gratuits étaient originaires de communes de plus de 2000 habitants, mais aussi que plus de la moitié d'entre eux résidaient dans les plus grandes villes de France. Paris et Strasbourg sont à elles seules à l'origine de plus de $30 \%$ des passages accordés. De la même façon, en dépit des remarques d'Alger sur les difficultés provoquées par la présence des classes laborieuses urbaines dans la colonie, les artisans $(35 \%)$ étaient aussi nombreux que les manœuvres (34\%) et bien plus représentés que les travailleurs du secteur agricole (19\%). De plus, les métiers du bâti-

40. Roger Price, 'Techniques of Repression: The Control of Popular Protest in Nineteenth-Century France', The Historical Journal, tome 25, no. 4, 1982, p. 859

41. Arch. dép. Ariège 10M 15, circulaire du ministre de la Guerre, 19 janvier 1855. 
ment, de la métallurgie et de services divers - plus précisément ceux qui "s'exercent de préférence dans les villes» - prédominaient largement. Les ouvriers du bâtiment et de la métallurgie étaient recrutés pour des ouvrages de travaux publics et, très logiquement, formaient $59 \%$ des bénéficiaires des permis de passage gratuit. Mais, au-delà, un éventail large de secteurs - alimentation, textile et habillement, produits de luxe - accueillait tout de même $25 \%$ des migrants assistés ${ }^{42}$.

Le portrait type du migrant assisté était bien loin du colon idéal envisagé par les promoteurs de la colonisation de peuplement en Algérie, c'est-à-dire un jeune paysan économe et robuste pouvant "se passer sans chagrin des sociétés nombreuses, et des divertissements qu'on ne trouve que dans les villes» et pour lequel «la vie de famille, la liberté, et les plaisirs ruraux, doivent suffire $»^{43}$. Les individus admis à recevoir des aides à l'émigration dans les années 1840 ressemblaient au contraire à ceux dont se plaignaient si souvent les autorités coloniales. Leur départ vers l'Algérie était précisément motivé par le désir de fuir une situation critique, sur le plan économique et familial. Mathieu Courbon, ouvrier soyeux sans emploi, habitant des Brotteaux à Lyon, aurait pu parler au nom de milliers d'autres lorsqu'il écrivait au préfet du Rhône en 1847 qu'il "désir[ait] aller en Algérie dans l'espoir d'y trouver du travail, fait de son état ou d'autre vu sa position malheureuse $[$ sic $]{ }^{44}$. Ce que les archives laissent voir des stratégies et des réseaux mobilisés par les migrants suggère que les politiques d'émigration et les relations interpersonnelles étaient des facteurs essentiels de l'émigration : les migrants répondaient à la double injonction de la publicité officielle et des appels de la famille ou des amis déjà installés en Afrique du Nord ${ }^{45}$.

Il faudrait poursuivre l'analyse pour obtenir une image plus précise des stratégies d'émigration coloniale et des expériences de migrants en Algérie. Des taux de mortalité élevés et un fort turnover suggèrent que pour beaucoup l'émigration n'aura été qu'une courte parenthèse. Nous savons encore très peu de choses des interactions entre les migrants français et la population locale en Algérie, ou des relations sociales à l'intérieur de la communauté européenne polyglotte durant la période de la conquête. Mais le caractère paradoxal de la politique d'émigration des premières années de la présence française est indiscutable. Faire de l'Algérie une colonie de peuplement apparaissait comme une solution aux difficultés de la France post-révolutionnaire, mais l'émigration d'individus perturbateurs représentait une menace pour un ordre colonial en formation. Ces difficultés, et le fait que les aides

42. Arch. nat. Outre-Mer, F80 *1998-*2000, Passages gratuits, 1841-1845. Ces données sont analysées de manière plus approfondie dans mon ouvrage à paraître, Empire of Virtue, op. cit.

43. Jean-Baptiste Say, Cours complet..., op. cit., p. 401.

44. Arch. mun. Lyon, 4WP 59 2, Mathieu Courbon au préfet du Rhône, vers 5 février 1847.

45. Arch. dép. Bas-Rhin, 3M 672, maire de Schweighausen au préfet du Bas-Rhin, 18 octobre 1838; Arch. mun. Lyon, 4WP 59 2, commissaire de police au maire de La Guillotière, 26 février 1845 . 
à l'émigration étaient considérées par les agents de l'État comme une forme d'assistance, ont conduit à définir les pauvres, les travailleurs migrants et les citadins comme des "indésirables» à exclure de la colonie. Mais l'idéal d'une colonisation agricole et familiale s'est trouvé en porte-à-faux avec les réalités de la guerre de conquête et de l'économie coloniale : une colonisation agricole à grande échelle exigeait une infrastructure civile et militaire que seuls des ouvriers professionnels, venant de milieux urbains, pouvaient bâtir. D'où le paradoxe d'une politique française d'émigration qui se donne pour tâche d'identifier, de recruter et de soutenir des migrants considérés comme indésirables. Ce paradoxe ne se limite d'ailleurs pas aux émigrants français : en dépit de la politique officielle favorisant l'émigration en provenance de métropole, le peu d'empressement des Français à s'installer en Algérie a conduit le ministère de la Guerre à étendre les programmes d'assistance à tous les Européens, quand ceux-ci furent mis en place en 1838. Mais en pratique, seuls les habitants de la Suisse et de la Rhénanie purent en bénéficier : le ministère voulait en effet favoriser ces populations aux dépens des Européens du Sud, bien plus nombreux en Algérie. La présence des Espagnols, Maltais et Italiens est donc le résultat d'initiatives privées et d'une immigration clandestine que les autorités portuaires et consulaires françaises avaient bien du mal à contrôler ${ }^{46}$. De fait, l'immigration méditerranéenne fut implicitement tolérée et l'encadrement administratif des flux de migrants a donc surtout concerné les travailleurs français.

Ce paradoxe d'une émigration assistée quoique indésirable est caractéristique de la période de conquête pendant laquelle les autorités étaient particulièrement sensibles à la précarité de la présence française en Algérie. Ce n'est qu'avec la fin de la conquête militaire en 1857 suivie de l'augmentation conséquente de la population européenne en Algérie, que les fondements de la politique d'émigration changèrent de nature. Si en 1848 le projet de colonies agricoles s'inscrit dans le programme républicain, la réglementation en vigueur n'a pas fondamentalement changé : l'arrivée de nouveaux colons suscite des conflits fort comparables à ceux qui ont opposé le général Berthezène aux autorités métropolitaines dix-huit ans plus tôt ${ }^{47}$. Le passeport ne cessa d'être obligatoire pour voyager entre la France et l'Algérie qu'en 1862, à un moment où, en France métropolitaine, les forces de police ne contrôlaient plus guère les passeports pour l'intérieur, même si le gouverneur général Pélissier continuait d'affirmer que «la tolérance accordée à ceux qui voyagent dans un but d'affaires, de science ou de plaisir, ne saurait s'étendre aux vagabonds, aux gens sans aveu, aux repris de justice, aux hommes dangereux de

46. Jennifer Sessions, "L'Algérie devenue française": The Naturalization of Non-French Colonists in French Algeria, 1830-1849', Proceedings of the Western Society for French History, tome 30 (2002), p. 169-171; Emile Temime, «La migration européenne...", op. cit., p. 37-38.

47. Yvette Katan, "Les colons de 1848...», loc. cit., p. 179; Michael Heffernan, 'The Parisian Poor and the Colonization of Algeria during the Second Republic,' French History, tome 3, nº. 4, 1989, p. $377-403$. 
toute espèce ${ }^{48}$. À la fin du siècle, les concessions agricoles étaient devenues le principal moyen d'encourager l'émigration coloniale et les passages gratuits ou subventionnés ne concernaient plus que les individus qui avaient reçu des concessions et répondaient aux cinq critères suivants : être français; être chef de famille; avoir des connaissances agricoles; disposer de ressources propres suffisantes pour développer la concession (au moins 5000 francs); s'engager à rester sur la concession pour au moins cinq $a s^{49}$.

Des conditions similaires avaient été imposées aux concessionnaires dès les premières cessions de terre dans le but d'éviter la spéculation foncière et de s'assurer que les propriétaires exploiteraient eux-mêmes leurs terres. Cet aspect de la politique coloniale n'avait donc rien de nouveau. Mais la limitation des passages gratuits aux colons-concessionnaires marqua une inflexion importante par rapport aux premières politiques d'émigration assistée qui visaient avant tout les colons-ouvriers. L'exclusion ultérieure des migrants de la classe ouvrière des programmes d'assistance souligne les particularités de la période de conquête, au cours de laquelle les impératifs de sécurité et de développement de l'infrastructure l'avaient emporté sur l'idéologie de la colonisation agricole. Avec l'instauration d'un régime civil et l'assimilation administrative à la France sous la Troisième République, les objectifs de la politique d'émigration prirent davantage en compte les nécessités de la colonisation dans les zones rurales. Les migrants issus de la classe ouvrière étaient maintenant libres de se rendre dans les départements algériens, mais à leurs frais. Dorénavant, le gouvernement n'offrirait son assistance qu'aux personnes habilitées à recevoir des concessions de terres et capables de contribuer utilement au développement de la colonisation rurale.

Jennifer Sessions est "assistant professor" à l'université de l'Iowa (The University of Iowa) Article traduit de l'anglais par Najate Zouggari

48. Bulletin officiel du Gouvernement Général de l’Algérie, 2 (1862), n 44, circulaire du Gouverneur-général, 2 avril 1862; Gérard Noiriel, «Surveiller les déplacements... », loc. cit., p. 95-98.

49. Arch. dép. Var 11M 3/1, Gouvernement Général de l'Algérie, Colonisation. Peuplement de nouveaux villages. Année 1902, Mustapha, Imprimerie algérienne, 1902, p. 1-2. 